\title{
Semiclassical transport equations of Dirac particles in rotating frames
}

\author{
Ömer F. Dayi® and Eda Kilinçarslan $\odot$ \\ Physics Engineering Department, Faculty of Science and Letters, \\ Istanbul Technical University, TR-34469 Maslak-Istanbul, Turkey
}

(Received 20 April 2020; accepted 3 August 2020; published 21 August 2020)

\begin{abstract}
We establish covariant semiclassical transport equations of massive spin-1/2 particles which are generated by the quantum kinetic equation modified by enthalpy current dependent terms. The purpose of modification is to take into account the noninertial properties due to the angular velocity of rotating frame which is equivalent to the fluid vorticity in the hydrodynamical approach. We present the equations satisfied by the Wigner function components and by studying their solutions in the semiclassical approximation we accomplish the transport equations.To acquire a three-dimensional kinetic theory, the relativistic kinetic equations in the comoving frame are integrated over the zeroth component of fourmomentum. The resulting vector and axial-vector currents are calculated at zero temperature. There exists another three-dimensional formulation of Dirac particles which correctly addresses the noninertial features of rotating coordinates. We review it briefly and obtain the mass corrections to the chiral vector and axialvector currents produced by this formulation.
\end{abstract}

DOI: $10.1103 /$ PhysRevD.102.045015

\section{INTRODUCTION}

Quark-gluon plasma created in heavy-ion collisions form a novel phase of nuclear matter. The constituent quarks and gluons are deconfined, where the quarks can be considered as massless, hence can be right- or left-handed. In the systems composed of chiral fermions collective behavior generates the vector and axial-vector current terms proportional to magnetic field known as the chiral magnetic effect [1-3] and the chiral separation effect [4,5]. In currents, there also exist terms which are proportional to the vorticity. They lead to the chiral vortical effect [6] and the local (spin) polarization effect [7-9]. In these effects magnetic field and the vorticity of fluids play similar roles. In heavy-ion collisions vortical effects generate the polarization of $\Lambda$ as measured in [10]. The dynamics of chiral fermions can be investigated by means of the covariant quantum kinetic equation in Minkowski space [11,12]. However, this transport equation lacks the similarity between the magnetic field and the vorticity. It depends explicitly on electromagnetic field strength but the vorticity is incorporated in it through the fluid velocity $[9,13,14]$ and the equilibrium distribution function $[15,16]$. Moreover, in the Wigner function approach noninertial effects like the Coriolis force, do not appear. To take into account vorticity

Published by the American Physical Society under the terms of the Creative Commons Attribution 4.0 International license. Further distribution of this work must maintain attribution to the author(s) and the published article's title, journal citation, and DOI. Funded by SCOAP ${ }^{3}$. from the start, in [17] we proposed and studied a modified quantum kinetic equation for chiral fermions.

To solve a transport equation as the initial-value problem to guarantee that the distribution function is acceptable, three-dimensional (3D) kinetic equation is preferred [18-20]. A methodical approach of introducing a 3D kinetic theory is to integrate the four-dimensional (4D) one over the zeroth component of four-momentum, $p_{0}$ $[19,21]$. To perform this calculation one should choose a frame, but not each possible choice yields an acceptable 3D kinetic theory. By integrating the 4D transport theory proposed in [17] we accomplished the 3D kinetic theory which is consistent with the chiral phenomena. It is the unique consistent 3D semiclassical chiral kinetic equation which does not depend explicitly on the 3D position vector $\boldsymbol{x}$, and possesses the Coriolis force. There exists a similar 3D formulation [22] which has been established from the relativistic theory given in curved spacetime [23] by integrating over $p_{0}$, but it depends on $\boldsymbol{x}$ explicitly. A different approach of defining relativistic kinetic equations and the related 3D transport theories were discussed in [24,25]. A consistent nonrelativistic kinetic formulation of chiral fermions both in the presence of electromagnetic fields and global rotations was given in [26] by working directly in 3D. Its starting point is the equivalence between fluid vorticity in hydrodynamical approach and angular velocity of fluid in the comoving frame [27]. The kinetic theory of [26] also depends position explicitly, but it possesses the characteristic feature of being acquired as the vanishing mass limit of the kinetic theory of Dirac particles. Furthermore, in this formulation similarity 
between the vorticity and magnetic field is explicit. Thus, it generates some new phenomena like the rotational analogue of Hall effect in nonlinear transport of chiral plasma [28].

Although in quark-gluon plasma the quarks are treated as massless, in reality they are massive. Thus it is crucial to understand how nonvanishing fermion mass affects the chiral transport phenomena. The quantum kinetic equation of Wigner function was originally introduced for massive Dirac particles. The Wigner function can be decomposed in terms of the Clifford algebra generators whose coefficients are scalar, pseudoscalar, vector, axial-vector and tensor fields. These fields satisfy a group of differential equations. In the massless case equations satisfied by the vector and axial-vector field components decouple from the rest. However, for massive fermions one can choose different sets of field components to construct kinetic equations. One of the possible choices was given in [29]. Another choice was presented in [30], where the vector and axial-vector fields were chosen as independent functions to construct kinetic equations whose massless limit can directly be acquired. An alternative way of constructing transport equations from the quantum kinetic equation was given in [31]. This approach has been extended to construct kinetic theory of massive fermions in curved spacetimes [32]. In the context of the semiclassical Wigner function method the study of massive fermions was started in $[33,34]$. By means of the solutions of Dirac equation they constructed Wigner function components at the zeroth-order in Planck constant. Then, inspired by the chiral one, they wrote the axial-vector field at the first-order in Planck constant and the properties of polarization as well as pseudoscalar condensation for massive fermions have been investigated. Mass corrections to chiral phenomena were also inspected by means of equaltime kinetic equations [35].

The aim of this work is to study massive spin- $1 / 2$ fermions in terms of two different semiclassical kinetic theory approaches whose common characteristics lie in the facts that the similarity between magnetic field and vorticity is manifest, and the noninertial features like the Coriolis force are addressed correctly. The first one is the 3D formulation presented in [26]. We study the vector and axial-vector currents by paying attention to the terms which are linear in the magnetic field and the angular velocity. We calculate mass corrections to chiral effects. The second one is a relativistic approach. We propose to modify the quantum kinetic equation of Dirac particles by means of some enthalpy current dependent terms guided by the chiral formalism [17]. But the modification of the quantum kinetic equation of Dirac particles differ from the massless case with the terms which vanish in the massless limit. To derive kinetic equations generated by the modified quantum kinetic equation we mainly follow the method of [31]. After accomplishing the kinetic equations of distribution functions we integrate them over $p_{0}$. Thereby we establish the 3D transport equations in the helicity basis. This formulation leads to the dispersion relation which is in accord with the one resulting from the Dirac Hamiltonian in rotating coordinates. Moreover, the Coriolis force is generated. We study mass corrections to chiral phenomena.

In Sec. II, the 3D formulation of [26] will be reviewed briefly. The resulting vector and axial-vector currents in the helicity basis will be presented. Then the mass corrections to magnetic and vortical effects will be calculated at zero temperature. In Sec. III, we discuss the modified quantum kinetic equation and obtain the transport equations. In comoving frame we integrate them over $p_{0}$ after choosing the quantization direction appropriately. Thereby the 3D kinetic equations are established. We discuss the particle number currents deduced from them at zero temperature. In the last section we present discussions of our results and open problems.

\section{3D SEMICLASSICAL KINETIC THEORY}

In [26] dynamical features of Dirac particles are studied by means of the semiclassical wave packets of freeparticles, $e=1$ and free-antiparticles, $e=-1$,

$$
\psi_{x^{\prime}}^{e}(\boldsymbol{p})=\sum_{\alpha=1,2} \xi_{\alpha}^{e} u_{\alpha}^{e}(\boldsymbol{p}, E) e^{-e i \boldsymbol{p} \cdot x^{\prime} / \hbar}
$$

where $\boldsymbol{x}^{\prime}$ is the 3D position variable and $\boldsymbol{x}, \boldsymbol{p}$ denote the phase-space coordinates of wave packet center. $E=\sqrt{\boldsymbol{p}^{2}+m^{2}}$, is the energy of free particle of mass $m$. The spinors $u_{\alpha}^{e}(\boldsymbol{p}, E)$ denote the four linearly independent solutions of the Dirac equation and $\xi_{\alpha}^{e}$ are some constant coefficients.

To expose the formulation of [26] briefly, let us suppress the index $e$, and deal with positive energy solutions. We define the one-form $\eta_{0}$ through

$$
\int\left[d x^{\prime}\right] \delta\left(\boldsymbol{x}-\boldsymbol{x}^{\prime}\right) \Psi_{x^{\prime}}^{\dagger}\left(-i \hbar d-H_{D} d t\right) \Psi_{x^{\prime}}=\sum_{\alpha \beta} \xi_{\alpha}^{*} \eta_{0 \alpha \beta} \xi_{\beta} .
$$

$\eta_{0}$ is a $2 \times 2$ matrix in "spin indices." It can be written as

$$
\eta_{0 \alpha \beta}=\delta_{\alpha \beta} \boldsymbol{p} \cdot d \boldsymbol{x}-\boldsymbol{A}_{\alpha \beta} \cdot d \boldsymbol{p}-H_{\alpha \beta}^{D} d t .
$$

$H_{\alpha \beta}^{D}$ is the projection of Dirac Hamiltonian on the particle solutions, $u_{\alpha} \equiv u_{\alpha}^{1}$, and

$$
\boldsymbol{A}_{\alpha \beta}=-i \hbar u_{\alpha}^{\dagger}(\boldsymbol{p}) \frac{\partial}{\partial \boldsymbol{p}} u_{\beta}(\boldsymbol{p})=\hbar \frac{\boldsymbol{\sigma}_{\alpha \beta} \times \boldsymbol{p}}{2 E(E+m)},
$$

is the matrix valued Berry gauge field where $\sigma$ are the Pauli spin matrices. To establish Hamiltonian formulation we define the following symplectic two-form by suppressing the matrix indices, 


$$
\zeta_{0 t}=d \eta_{0} \equiv d p_{i} \wedge d x_{i}-G-D_{i} H_{D} d p_{i} \wedge d t
$$

The repeated indices $i, j=1,2,3$, are summed over. We introduced the covariant derivative:

$$
\boldsymbol{D} \equiv \frac{\partial}{\partial \boldsymbol{p}}+\frac{i}{\hbar}[\boldsymbol{A},]
$$

Unit matrices are not explicitly written. $G=\frac{1}{2} G_{i j} d p_{i} \wedge$ $d p_{j}$ is the Berry curvature two-form which can be expressed in terms of the vector $\boldsymbol{G}$ as

$$
G_{i j}=\frac{\partial A_{j}}{\partial p_{i}}-\frac{\partial A_{i}}{\partial p_{j}}+\frac{i}{\hbar}\left[A_{i}, A_{j}\right]=\epsilon_{i j k} G_{k} .
$$

By plugging (4) into (7) one obtains

$$
\boldsymbol{G}=\frac{\hbar m}{2 E^{3}}\left(\boldsymbol{\sigma}+\frac{\boldsymbol{p}(\boldsymbol{\sigma} \cdot \boldsymbol{p})}{m(m+E)}\right) .
$$

Up to now we dealt with the free Dirac particles. To discuss the electromagnetic interactions and the rotation of reference frame one needs to insert the appropriate gauge fields into the one-form. Once this is done the symplectic twoform matrix can be established as

$$
\begin{aligned}
\zeta_{t}= & d p_{i} \wedge d x_{i}+\frac{1}{2} \epsilon_{i j k}\left(q B_{k}+2 \mathcal{E} \omega_{k}\right) d x_{i} \wedge d x_{j} \\
& -\frac{1}{2} \epsilon_{i j k} G_{k} d p_{i} \wedge d p_{j}+\epsilon_{i j k} x_{j} \omega_{k} \nu_{m} d x_{i} \wedge d p_{m} \\
& -\nu_{i} d p_{i} \wedge d t+\frac{1}{2} \nu_{i}(\boldsymbol{\omega} \times \boldsymbol{x})^{2} d p_{i} \wedge d t \\
& +[q \boldsymbol{E}+(\boldsymbol{\omega} \times \boldsymbol{x}) \times(q \boldsymbol{B}+\mathcal{E} \boldsymbol{\omega})]_{i} d x_{i} \wedge d t .
\end{aligned}
$$

The dispersion relation $\mathcal{E}$ and the related velocity $\boldsymbol{v}$ will be written explicitly in the sequel. $\boldsymbol{E}, \boldsymbol{B}$ are the electromagnetic fields and $\boldsymbol{\omega}$ is the angular velocity of the rotating frame. We consider nonrelativistic global rotations, thus the linear velocity is bounded: $|\boldsymbol{\omega} \times \boldsymbol{x}| \ll c$.

To establish the semiclassical transport equations consider the volume form

$$
\begin{aligned}
\Omega & =\frac{1}{3 !} \zeta_{t} \wedge \zeta_{t} \wedge \zeta_{t} \wedge d t \\
& =\frac{1}{3 !} \zeta \wedge \zeta \wedge \zeta \wedge d t
\end{aligned}
$$

The matrix valued $\left.\zeta \equiv \zeta_{t}\right|_{d t=0}$ is the symplectic two-form in the phase-space given by $(\boldsymbol{x}, \boldsymbol{p})$. One can equivalently write (10) as

$$
\Omega=\zeta_{1 / 2} d V \wedge d t
$$

$d V$ is the canonical volume form and the phase space measure $\zeta_{1 / 2}$ is the Pfaffian of the $(6 \times 6)$ matrix,

$$
\left(\begin{array}{cc}
\epsilon_{i j k}\left(q B_{k}+2 E \omega_{k}\right) & -\delta_{i j}+\nu_{j}(\boldsymbol{x} \times \boldsymbol{\omega})_{i} \\
\delta_{i j}-\nu_{i}(\boldsymbol{x} \times \boldsymbol{\omega})_{j} & -\epsilon_{i j k} G_{k}
\end{array}\right) .
$$

To attain the Liouville equation let us introduce $i_{v}$, which is the interior product of the vector field

$$
v=\frac{\partial}{\partial t}+\dot{\boldsymbol{x}} \frac{\partial}{\partial \boldsymbol{x}}+\dot{\boldsymbol{p}} \frac{\partial}{\partial \boldsymbol{p}} .
$$

Note that $v$ and the time evolutions $(\dot{\boldsymbol{x}}, \dot{\boldsymbol{p}})$ are matrixvalued. As usual, we suppressed the unit matrix. The Lie derivative $\mathcal{L}_{v}=i_{v} d+d i_{v}$, of the volume form yields the Liouville equation:

$$
\mathcal{L}_{v} \Omega=0
$$

The Lie derivative can be calculated in two different ways. By comparing them one acquires the following relation

$$
\left(\frac{\partial \zeta_{1 / 2}}{\partial t}+\frac{\partial}{\partial \boldsymbol{x}} \cdot\left(\dot{\boldsymbol{x}} \zeta_{1 / 2}\right)+\boldsymbol{D} \cdot\left(\zeta_{1 / 2} \dot{\boldsymbol{p}}\right)\right) d V \wedge d t=\frac{1}{3 !} d \zeta_{t}{ }^{3}
$$

Calculation of $\zeta_{t}^{3}$ yields the explicit form of Pfaffian and the time evolution of phase space variables. Similar calculations can be carried out for antiparticles. Hence, by retrieving the particle/antiparticle index $e$, we get

$$
\begin{aligned}
\zeta_{1 / 2}^{e}= & 1+\boldsymbol{G}^{e} \cdot\left(e \boldsymbol{B}+2 \mathcal{E}^{e} \boldsymbol{\omega}\right)-\boldsymbol{\nu}^{e} \cdot(\boldsymbol{x} \times \boldsymbol{\omega}) \\
& -\left(\boldsymbol{\nu}^{e} \cdot \boldsymbol{G}^{e}\right)[e \boldsymbol{B} \cdot(\boldsymbol{x} \times \boldsymbol{\omega})], \\
\dot{\boldsymbol{x}}^{e} \zeta_{1 / 2}^{e}= & \boldsymbol{\nu}^{e}\left[1-\frac{1}{2}(\boldsymbol{\omega} \times \boldsymbol{x})^{2}\right]+\boldsymbol{E}_{\omega}^{e} \times \boldsymbol{G}^{e} \\
& +\left(\boldsymbol{\nu}^{e} \cdot \boldsymbol{G}^{e}\right)\left(e \boldsymbol{B}+2 \mathcal{E}^{e} \boldsymbol{\omega}\right)\left[1-\frac{1}{2}(\boldsymbol{\omega} \times \boldsymbol{x})^{2}\right] \\
& +\left(\boldsymbol{\nu}^{e} \cdot \boldsymbol{G}^{e}\right)\left[(\boldsymbol{x} \times \boldsymbol{\omega}) \times \boldsymbol{E}_{\omega}^{e}\right], \\
\zeta_{1 / 2}^{e} \dot{\boldsymbol{p}}^{e}= & \boldsymbol{E}_{\omega}^{e}+\boldsymbol{\nu}^{e} \times\left(e \boldsymbol{B}+2 \mathcal{E}^{e} \boldsymbol{\omega}\right)\left[1-\frac{1}{2}(\boldsymbol{\omega} \times \boldsymbol{x})^{2}\right] \\
& +\boldsymbol{G}^{e}\left[\boldsymbol{E}_{\omega}^{e} \cdot\left(e \boldsymbol{B}+2 \mathcal{E}^{e} \boldsymbol{\omega}\right)\right]-\left[(\boldsymbol{x} \times \boldsymbol{\omega}) \times \boldsymbol{E}_{\omega}^{e}\right] \times \boldsymbol{\nu}^{e} .
\end{aligned}
$$

We introduced $\boldsymbol{G}^{e}=e \boldsymbol{G}$, and the effective electric fields

$$
\boldsymbol{E}_{\omega}^{e}=e \boldsymbol{E}+(\boldsymbol{\omega} \times \boldsymbol{x}) \times\left(e \boldsymbol{B}+\mathcal{E}^{e} \boldsymbol{\omega}\right) .
$$

We set the electric charge to unity, so that $q=e$. The dispersion relation and the related velocity are obtained from the Hamiltonian of charged Dirac particle under the influence of external electromagnetic fields, in a rotating frame as 


$$
\mathcal{E}^{e}=E\left[1-\boldsymbol{G}^{e} \cdot(e \boldsymbol{B}+E \boldsymbol{\omega})\right]
$$$$
\boldsymbol{\nu}^{\boldsymbol{e}}=\frac{\boldsymbol{p}}{E}\left[1+\boldsymbol{G}^{e} \cdot(2 e \boldsymbol{B}+E \boldsymbol{\omega})\right]-\frac{\hbar \chi e}{2 E^{3}}(e \boldsymbol{B}+E \boldsymbol{\omega}) \boldsymbol{\sigma} \cdot \boldsymbol{p} .
$$

Distribution functions are needed to define particle number and current densities from one-particle quantities. Within this formalism distribution functions are matrices whose elements are $f_{\alpha \beta}^{e}$. In principle all of the four elements can be nonvanishing. However, we are mainly interested in mass corrections to the chiral kinetic theory. Hence, it is convenient to work in a basis which permits us to choose the distribution function to be diagonal:

$$
f^{e}=\left(\begin{array}{cc}
f_{1}^{e} & 0 \\
0 & f_{-1}^{e}
\end{array}\right)
$$

For massless fermions $f_{\chi}^{e} ; \chi=1,-1$, coincide with distribution functions of the right- and left-handed particles/ antiparticles. This basis can be called as the helicity basis: Helicity matrix is given by

$$
\lambda=\frac{\boldsymbol{\Sigma} \cdot \boldsymbol{p}}{|\boldsymbol{p}|}
$$

where $\boldsymbol{\Sigma}=\left(\begin{array}{ll}\boldsymbol{\sigma} & 0 \\ 0 & \sigma\end{array}\right)$ is the spin matrix. One can show that for the free particle/antiparticle solutions

$$
u_{\alpha}^{e \dagger} \Sigma u_{\beta}^{e}=e \boldsymbol{\sigma}_{\alpha \beta} .
$$

Recall that each $u_{\alpha}^{e}$ is a 4-spinor. Now, let us change the basis by the $2 \times 2$ matrix $R$ which is defined to satisfy

$$
R^{\dagger} \boldsymbol{\sigma} R=\left(\begin{array}{cc}
1 & 0 \\
0 & -1
\end{array}\right) \boldsymbol{n}
$$

where $\boldsymbol{n}$ is the spin quantization direction in the rest frame of particle. Observe that by choosing

$$
\boldsymbol{n}=\hat{\boldsymbol{p}},
$$

we can diagonalize the helicity matrix:

$$
\left(u^{e} R\right)^{\dagger}\left(\frac{\boldsymbol{\Sigma} \cdot \boldsymbol{p}}{|\boldsymbol{p}|}\right)\left(u^{e} R\right)=\left(\begin{array}{cc}
1 & 0 \\
0 & -1
\end{array}\right) .
$$

Hence the choice (25) is in accord with the helicity basis. In this basis all the matrix valued quantities obtained above are diagonal. $\chi$ labels the diagonal elements. For example the Berry curvature (8) is now diagonal whose elements are

$$
\boldsymbol{G}_{\chi}^{e}=\frac{\hbar e \chi \hat{\boldsymbol{p}}}{2 E^{2}}
$$

Thus, the dispersion relation (19) turns out to be

$$
\mathcal{E}_{e}^{\chi}=E-\frac{\hbar e \chi \hat{\boldsymbol{p}}}{2 E} \cdot(e \boldsymbol{B}+E \boldsymbol{\omega}) .
$$

The vorticity dependent shift in dispersion relation (28) is independent of mass. For chiral particles the same term was obtained in $[36,23]$.

We introduce the collisionless transport equations as

$$
\zeta_{e}^{\chi} \frac{\partial f_{\chi}^{e}}{\partial t}+\dot{\boldsymbol{x}}_{e}^{\chi} \zeta_{e}^{\chi} \cdot \frac{\partial f_{\chi}^{e}}{\partial \boldsymbol{x}}+\zeta_{e}^{\chi} \dot{\boldsymbol{p}}_{e}^{\chi} \cdot \frac{\partial f_{\chi}^{e}}{\partial \boldsymbol{p}}=0 .
$$

Now one can define particle number and current densities as

$$
\begin{gathered}
n_{\chi}(\boldsymbol{x}, t)=\int \frac{d^{3} p}{(2 \pi \hbar)^{3}} \sum_{e} \zeta_{e}^{\chi} f_{\chi}^{e}, \\
\boldsymbol{j}_{\chi}(\boldsymbol{x}, t)=\int \frac{d^{3} p}{(2 \pi \hbar)^{3}} \sum_{e}\left(\dot{\boldsymbol{x}}_{e}^{\chi} \zeta_{e}^{\chi}\right) f_{\chi}^{e} .
\end{gathered}
$$

By setting $\int \frac{d^{3} p}{(2 \pi \hbar)^{3}} \sum_{e} \partial_{p} \cdot\left(\zeta_{e}^{\chi} \dot{\boldsymbol{p}}_{e}^{\chi} f_{\chi}^{e}\right)=0$, the continuity equation follows,

$$
\frac{\partial n_{\chi}(\boldsymbol{x}, t)}{\partial t}+\boldsymbol{\nabla} \cdot \boldsymbol{j}_{e}(\boldsymbol{x}, t)=0
$$

The vector and axial vector currents are defined as

$$
\boldsymbol{j}_{V}(\boldsymbol{x}, t)=\sum_{\chi} \boldsymbol{j}_{\chi}(\boldsymbol{x}, t), \quad \boldsymbol{j}_{A}(\boldsymbol{x}, t)=\sum_{\chi} \chi \boldsymbol{j}_{\chi}(\boldsymbol{x}, t)
$$

They possess linear terms in $\boldsymbol{B}$ and $\boldsymbol{\omega}$ which can be expressed by

$$
\boldsymbol{j}_{A, V}^{B, \omega}(\boldsymbol{x}, t)=\sigma_{A, V}^{B} \boldsymbol{B}+\sigma_{A, V}^{\omega} \boldsymbol{\omega}
$$

Let us choose the equilibrium distribution function as the Fermi-Dirac distribution:

$$
f_{(\mathcal{E}) \chi}^{F D e}=\frac{1}{e^{e\left[\mathcal{E}_{e}^{\chi}-\mu_{\chi}\right] / T}+1}
$$

After performing the angular parts of the momentum space integrals we acquire

$$
\begin{gathered}
\sigma_{A, V}^{B}=\frac{1}{6 \pi^{2} \hbar^{2}} \int d|\boldsymbol{p}|\left\{\frac{|\boldsymbol{p}|^{3}}{E^{3}} f_{A, V}^{F D}-\frac{|\boldsymbol{p}|^{3}}{2 E^{2}} \frac{\partial f_{A, V}^{F D}}{\partial E}\right\}, \\
\sigma_{A, V}^{\omega}=\frac{1}{3 \pi^{2} \hbar^{2}} \int d|\boldsymbol{p}|\left\{\frac{|\boldsymbol{p}|^{3}}{E^{2}} f_{A, V}^{F D}-\frac{|\boldsymbol{p}|^{3}}{4 E} \frac{\partial f_{A, V}^{F D}}{\partial E}\right\},
\end{gathered}
$$

where, after relabeling $\mu_{1} \equiv \mu_{R}$ and $\mu_{-1} \equiv \mu_{L}$,

$$
f_{A, V}^{F D}=\sum_{e}\left\{\frac{1}{e^{e\left[E-\mu_{R}\right] / T}+1} \pm \frac{1}{e^{e\left[E-\mu_{L}\right] / T}+1}\right\} .
$$


The upper and lower signs correspond to axial-vector and vector currents, respectively. These produce the correct results for $m=0$, as they have been calculated explicitly in [28]. For simplicity let us deal with $\mu_{R}=\mu_{L}=\mu$. Then, the vector current coefficients vanish: $\sigma_{V}^{B, \omega}=0$. By performing the integrals in (36) and (37) at zero temperature one gets

$$
\begin{aligned}
\sigma_{A}^{B} & =\frac{1}{2 \pi^{2} \hbar^{2}}\left(\frac{\mu^{2}+3 m^{2}}{\mu}-\frac{4}{3} m\right) \theta(\mu-m), \\
\sigma_{A}^{\omega} & =\frac{1}{2 \pi^{2} \hbar^{2}}\left(\mu^{2}-m^{2}-\frac{4}{3} m^{2} \ln \left(\frac{\mu}{m}\right)\right) \theta(\mu-m) .
\end{aligned}
$$

For small mass we may approximately set $\boldsymbol{\nu} \approx \hat{\boldsymbol{p}}$ and deal with $f_{A, V}^{F D}$, from the start. At $T=0$, this approximation yields

$$
\begin{aligned}
\sigma_{A}^{B} & =\frac{1}{2 \pi^{2} \hbar^{2}} \int_{m}^{\mu} d E \frac{\sqrt{E^{2}-m^{2}}}{E} \\
& \approx \frac{1}{2 \pi^{2} \hbar^{2}} \sqrt{\mu^{2}-m^{2}} \theta(\mu-m),
\end{aligned}
$$

and

$$
\begin{aligned}
\sigma_{A}^{\omega} & =\frac{1}{\pi^{2} \hbar^{2}} \int_{m}^{\mu} d E \sqrt{E^{2}-m^{2}} \\
& \approx \frac{\mu}{2 \pi^{2} \hbar^{2}} \sqrt{\mu^{2}-m^{2}} \theta(\mu-m) .
\end{aligned}
$$

These are in accord with the results acquired by field theoretic calculations using Kubo formula for Dirac particles [37-39]. Although for $T \neq 0$ it seems that small mass corrections differ, a detailed study is needed.

\section{MODIFIED QUANTUM KINETIC EQUATION}

Quantum kinetic equation of relativistic fluids has been derived for charged Dirac particles in the presence of external electromagnetic fields [11,12]. It explicitly depends on the electromagnetic field strength $F_{\mu \nu}$. But the vorticity tensor of fluid which plays a role similar to electromagnetic field strength, shows up through the solution of kinetic equation. However, (non)inertial features of relativistic vorticity can be inspected by means of the circulation tensor:

$$
W_{\mu \nu}=\partial_{\mu} W_{\nu}-\partial_{\nu} W_{\mu}
$$

where $W_{\mu}$ is the enthalpy current and $\partial_{\mu} \equiv \partial / \partial x^{\mu}$. The enthalpy current is defined as $W_{\mu}=h u_{\mu}$, where $u_{\mu}=$ $d x_{\mu} / d \tau$ is the fluid four-velocity in the comoving frame and $h$ is the internal energy (enthalpy). Thus, the circulation tensor (41) turns out to be

$$
W_{\mu \nu}=h\left(\partial_{\mu} u_{\nu}-\partial_{\nu} u_{\mu}\right)+\left(\partial_{\mu} h\right) u_{\nu}-\left(\partial_{\nu} h\right) u_{\mu},
$$

In the rest frame of massive particles $h=m$, so that in [17] we proposed to modify the quantum kinetic equation of massless particles by the substitution:

$$
F_{\mu \nu} \rightarrow F_{\mu \nu}+W_{\mu \nu}-h\left(\partial_{\mu} u_{\nu}-\partial_{\nu} u_{\mu}\right)
$$

By virtue of the modified relativistic formulation we established a 3D semiclassical chiral kinetic theory which is consistent with anomalous chiral effects. Moreover, it possesses the Coriolis force and does not depend explicitly on the 3D position vector $\boldsymbol{x}$. As we mentioned in the Introduction, it is the unique 3D theory which possesses all of these properties.

One can also incorporate the noninertial effects in the quantum kinetic equation by considering it in curved spacetime $[23,32]$. However, this is not in conflict with considering the modification (43) which is in Minkowski spacetime. In fact, in [22] we showed that the modified chiral theory can be extended to curved spacetime. However, depending on the chosen frame and observer, the modification terms may not give contribution to kinetic equation. We have shown that the formalism of [23] yields a consistent $3 \mathrm{D}$ chiral kinetic theory possessing Coriolis force but the vorticity terms depend explicitly on $\boldsymbol{x}$. Thus, studying noninertial effects in Minkowski spacetime by introducing modifications in terms of enthalpy current has its own virtues.

To incorporate the noninertial features of fluid vorticity into the Wigner function formalism of Dirac particles, we would like to modify their quantum kinetic equation in a similar manner. However, the modification of quantum kinetic equation for massive particles differs from the chiral case with some terms which should survive for massive particles. We will show that the proposed modification generates correctly the Coriolis force and the dispersion relation of the Dirac particle coupled to electromagnetic fields in a rotating frame.

We present the modified quantum kinetic equation in a frame moving with the four-velocity $v_{\mu} ; v_{\mu} v^{\mu}=1$, whose linear acceleration vanishes: $v_{\nu} \partial^{\nu} v_{\mu}=0$. We would like to modify the quantum kinetic equation with frame dependent terms adequate to consider the noninertial features of relativistic vorticity. Thus we propose

$$
\left[\gamma_{\mu}\left(\pi^{\mu}+\frac{i \hbar}{2} D^{\mu}\right)-m\right] W(x, p)=0,
$$

as the quantum kinetic equation in a rotating frame of reference with

$$
\begin{aligned}
D^{\mu} & \equiv \partial^{\mu}-j_{0}(\Delta)\left[F^{\mu \nu}+w^{\mu \nu}\right] \partial_{p \nu} \\
\pi^{\mu} & \equiv p^{\mu}-\frac{\hbar}{2} j_{1}(\Delta)\left[F^{\mu \nu}+w^{\mu \nu}\right] \partial_{p \nu}
\end{aligned}
$$


where $\partial_{p}^{\mu} \equiv \partial / \partial p_{\mu}$, and

$$
w_{\mu \nu}=\frac{h}{2}\left(\partial_{\mu} v_{\nu}-\partial_{\nu} v_{\mu}\right)+\left(\partial_{\mu} h\right) v_{\nu}-\left(\partial_{\nu} h\right) v_{\mu} .
$$

It is worth noting that $w_{\mu \nu}$ is not the full circulation tensor (41). As we will show, our choice is dictated by the fact that the correct dispersion relation and Coriolis force should be generated in 3D kinetic theory.

In this section the electric charge $e$ is suppressed. $j_{0}(x)$ and $j_{1}(x)$ are spherical Bessel functions in $\Delta \equiv \frac{\hbar}{2} \partial_{p} \cdot \partial_{x}$. The space-time derivative $\partial_{\mu}$ contained in $\Delta$ acts on $\left[F^{\mu \nu}+w^{\mu \nu}\right]$, but not on the Wigner function. In contrary $\partial_{p \nu}$ acts on the Wigner function, but not on $\left[F^{\mu \nu}+w^{\mu \nu}\right]$.

The decomposition of the Wigner function is written through the 16 generators of the Clifford algebra as

$W=\frac{1}{4}\left(\mathcal{F}+i \gamma^{5} \mathcal{P}+\gamma^{\mu} \mathcal{V}_{\mu}+\gamma^{5} \gamma^{\mu} \mathcal{A}_{\mu}+\frac{1}{2} \sigma^{\mu \nu} \mathcal{S}_{\mu \nu}\right)$,

where the coefficients $\mathcal{C} \equiv\left\{\mathcal{F}, \mathcal{P}, \mathcal{V}_{\mu}, \mathcal{A}_{\mu}, \mathcal{S}_{\mu \nu}\right\}$, respectively, are the scalar, pseudoscalar, vector, axial-vector, and antisymmetric tensor components of the Wigner function. These fields can be expanded in powers of Planck constant:

$$
\mathcal{C}=\sum_{n} \hbar^{n} \mathcal{C}^{(n)}
$$

We deal with the semiclassical approximation where only the zeroth- and first-order fields are considered. Thus, to derive the equations which they satisfy, instead of (45), (46), we only need to deal with

$$
\begin{gathered}
\nabla^{\mu} \equiv \partial_{x}^{\mu}-\left[F^{\mu \nu}+w^{\mu \nu}\right] \partial_{p \nu} \\
\Pi^{\mu} \equiv p^{\mu}-\frac{\hbar^{2}}{12}\left[\partial^{\alpha} F^{\mu \nu}+\partial^{\alpha} w^{\mu \nu}\right] \partial_{p \alpha} \partial_{p \nu} .
\end{gathered}
$$

By plugging the decomposed Wigner function (48) into the modified quantum kinetic equation (44), one derives the equations satisfied by the fields $\mathcal{C}$, whose real parts are

$$
\begin{gathered}
\Pi \cdot \mathcal{V}-m \mathcal{F}=0, \\
\Pi_{\mu} \mathcal{F}-\frac{\hbar}{2} \nabla^{\nu} \mathcal{S}_{\nu \mu}-m \mathcal{V}_{\mu}=0, \\
-\frac{\hbar}{2} \nabla_{\mu} \mathcal{P}+\frac{1}{2} \epsilon_{\mu \nu \alpha \beta} \Pi^{\nu} S^{\alpha \beta}+m \mathcal{A}_{\mu}=0, \\
\frac{\hbar}{2} \nabla_{[\mu} \mathcal{V}_{\nu]}-\epsilon_{\mu \nu \alpha \beta} \Pi^{\alpha} \mathcal{A}^{\beta}-m \mathcal{S}_{\mu \nu}=0, \\
\frac{\hbar}{2} \nabla \cdot \mathcal{A}+m \mathcal{P}=0
\end{gathered}
$$

and the imaginary parts are

$$
\begin{gathered}
\hbar \nabla \cdot \mathcal{V}=0, \\
\Pi \cdot \mathcal{A}=0, \\
\frac{\hbar}{2} \nabla_{\mu} \mathcal{F}+\Pi^{\nu} \mathcal{S}_{\nu \mu}=0, \\
\Pi_{\mu} \mathcal{P}+\frac{\hbar}{4} \epsilon_{\mu \nu \alpha \beta} \nabla^{\nu} \mathcal{S}^{\alpha \beta}=0, \\
\Pi_{[\mu} \mathcal{V}_{\nu]}+\frac{\hbar}{2} \epsilon_{\mu \nu \alpha \beta} \nabla^{\alpha} \mathcal{A}^{\beta}=0 .
\end{gathered}
$$

Some of these equations can be employed to express a portion of the fields in terms of the others. Hence, depending on the choice of independent set of fields one can follow different routes of studying the transport equations. Whatever the choices are, in the semiclassical approach one should first discuss the general solutions at the zeroth-order in Planck constant and then at the higher orders.

\section{A. General solutions at $\mathcal{O}(\hbar)$}

Employing the solutions of Dirac equation, $u_{\chi}^{e}(\boldsymbol{p}, E)$, the Wigner function components at the zeroth-order in Planck constant have been obtained in $[31,33,34]$ as follows

$$
\begin{aligned}
\mathcal{F}^{(0)} & =m \delta\left(p^{2}-m^{2}\right) f_{V}^{0}, \\
\mathcal{V}_{\mu}^{(0)} & =p_{\mu} \delta\left(p^{2}-m^{2}\right) f_{V}^{0}, \\
\mathcal{A}_{\mu}^{(0)} & =m s_{\mu} \delta\left(p^{2}-m^{2}\right) f_{A}^{0}, \\
\mathcal{S}_{\mu \nu}^{(0)} & =\Sigma_{\mu \nu} \delta\left(p^{2}-m^{2}\right) f_{A}^{0}, \\
\mathcal{P}^{(0)} & =0,
\end{aligned}
$$

where

$$
\Sigma_{\mu \nu}(x, p)=-\frac{1}{m} \epsilon_{\mu \nu \alpha \beta} p^{\alpha} s^{\beta},
$$

is the dipole-moment tensor. Obviously, (62) solve (52)-(61) by ignoring $\hbar$-dependent terms. We choose the distribution functions in accord with (21), so that the scalar functions $f_{V}^{0}, f_{A}^{0}$ are given as

$$
\begin{aligned}
& f_{V}^{0}(x, p) \equiv 2 \sum_{e \chi} \theta\left(e p^{0}\right) f_{\chi}^{e}(x, p), \\
& f_{A}^{0}(x, p) \equiv 2 \sum_{e \chi} s \theta\left(e p^{0}\right) f_{\chi}^{e}(x, p) .
\end{aligned}
$$


$s^{\mu}=\sum_{e} \theta\left(e p_{0}\right) s_{e}^{\mu}\left(\boldsymbol{p}, \boldsymbol{n}_{e}\right)$, is the spin quantization direction four-vector satisfying $s^{2}=-1, p \cdot s=0$. It can be written in terms of the spin quantization direction in the rest frame of particle (antiparticle) $\boldsymbol{n}_{e}$, as [40]

$$
s_{e}^{\mu}\left(\boldsymbol{p}, \boldsymbol{n}_{e}\right)=\left(\frac{\boldsymbol{p} \cdot \boldsymbol{n}_{e}}{m}, e \boldsymbol{n}_{e}+\frac{e \boldsymbol{p} \cdot \boldsymbol{n}_{e}}{m(E+m)} \boldsymbol{p}\right) .
$$

Observe that the dipole-moment tensor satisfies $\Sigma^{\mu \nu} \Sigma_{\mu \nu}=2$, and the axial-vector component can be expressed as

$$
\mathcal{A}_{\mu}^{(0)}=-\frac{1}{2} \epsilon_{\mu \nu \alpha \beta} p^{\nu} \Sigma^{\alpha \beta} f_{A}^{0} \delta\left(p^{2}-m^{2}\right) .
$$

Multiplying (52), (53), respectively, with $m, p_{\mu}$, and keeping the $\mathcal{O}(\hbar)$ terms yield

$$
\left(p^{2}-m^{2}\right) \mathcal{F}^{(1)}=\frac{1}{2}\left(F^{\mu \nu}+w^{\mu \nu}\right) \mathcal{S}_{\mu \nu}^{(0)} .
$$

Thus the scalar component of the Wigner function at the first-order in $\hbar$, can be written as

$$
\mathcal{F}^{(1)}=m \delta\left(p^{2}-m^{2}\right)\left(f_{V}^{1}+\frac{F^{\mu \nu}+w^{\mu \nu}}{2\left(p^{2}-m^{2}\right)} \mathcal{S}_{\mu \nu}^{(0)} f_{A}^{0}\right),
$$

where $f_{V}^{1}$ is a scalar function.

Multiplying (54), (55), respectively, with $\epsilon^{\mu \sigma \gamma \kappa} p_{\sigma}, m$ and joining them by employing (58), (59), (62), leads to

$$
\left(p^{2}-m^{2}\right) S^{\gamma \kappa(1)}=\left(F^{\gamma \kappa}+w^{\gamma \kappa}\right) \mathcal{F}^{(0)} .
$$

It is solved by

$$
S^{\gamma \kappa(1)}=m \delta\left(p^{2}-m^{2}\right)\left(\Sigma^{1 \gamma \kappa}+\frac{F^{\gamma \kappa}+w^{\gamma \kappa}}{\left(p^{2}-m^{2}\right)} f_{V}^{0}\right),
$$

where $\Sigma_{\gamma \kappa}^{1}$ can be thought of as the $\hbar$-order contribution to dipole-moment tensor. It satisfies the constraint equation which follows from (59) by keeping terms at $\mathcal{O}(\hbar)$ :

$$
\delta\left(p^{2}-m^{2}\right) p^{\kappa} \Sigma_{\gamma \kappa}^{1}=\frac{1}{2} \delta\left(p^{2}-m^{2}\right) \nabla_{\gamma} f_{V}^{0} .
$$

By substituting the axial-vector with (67) in (56) one finds

$$
\mathcal{P}^{(1)}=\frac{1}{4 m} \epsilon^{\mu \nu \alpha \beta} \nabla_{\mu}\left[p_{\nu} \Sigma_{\alpha \beta} f_{A}^{0} \delta\left(p^{2}-m^{2}\right)\right] .
$$

To acquire the vector field $\mathcal{V}_{\mu}$ at the $\hbar$-order, we insert (62) and (69) into (53):

$$
\begin{aligned}
\mathcal{V}_{\mu}^{(1)}= & \frac{p_{\mu}}{m} \mathcal{F}^{(1)}-\frac{\hbar}{2 m} \nabla^{\nu} S_{\mu \nu}^{(0)}, \\
= & \delta\left(p^{2}-m^{2}\right)\left[p_{\mu} f_{V}^{1}+\frac{1}{2} \nabla^{\nu}\left(\Sigma_{\mu \nu} f_{A}^{0}\right)\right], \\
& -\delta^{\prime}\left(p^{2}-m^{2}\right)\left[\frac{1}{2} p_{\mu}\left(F^{\alpha \beta}+w^{\alpha \beta}\right) \Sigma_{\alpha \beta}\right. \\
& \left.+\Sigma_{\mu \nu}\left(F^{\nu \alpha}+w^{\nu \alpha}\right) p_{\alpha}\right] f_{A}^{0} .
\end{aligned}
$$

By plugging (71) into (54) one gets the axial vector field at $\mathcal{O}(\hbar)$, as

$$
\begin{aligned}
\mathcal{A}_{\mu}^{(1)}= & \frac{\hbar}{2 m} \nabla_{\mu} \mathcal{P}^{(0)}-\frac{1}{2 m} \epsilon_{\mu \nu \alpha \beta} p^{\nu} S^{\alpha \beta(1)}, \\
= & -\frac{1}{2} \epsilon_{\mu \nu \alpha \beta} p^{\nu} \Sigma^{1 \alpha \beta} \delta\left(p^{2}-m^{2}\right) \\
& +\left(\tilde{F}_{\mu \nu}+\tilde{w}_{\mu \nu}\right) p^{\nu} f_{V}^{0} \delta^{\prime}\left(p^{2}-m^{2}\right),
\end{aligned}
$$

where $\tilde{F}_{\mu \nu}=\frac{1}{2} \epsilon_{\mu \nu \alpha \beta} F^{\alpha \beta}$ and $\tilde{w}_{\mu \nu}=\frac{1}{2} \epsilon_{\mu \nu \alpha \beta} w^{\alpha \beta}$, are the dual tensors.

We solved a part of the equations (52)-(61) to write the Wigner function components up to $\hbar$-order in terms of the undetermined distribution functions $f_{V}, f_{A}$ and spin quantization direction $\boldsymbol{n}$. Now, by using the rest of (52)-(61), we will establish the kinetic equations which they satisfy.

\section{B. Semiclassical kinetic equations}

By expanding (57) and (61) up to the first-order in $\hbar$, one derives the kinetic equations [31]:

$$
\begin{gathered}
\delta\left(p^{2}-m^{2}\right) p \cdot \nabla f_{V}^{0}=0, \\
\delta\left(p^{2}-m^{2}\right) p \cdot \nabla f_{A}^{0}=0, \\
\delta\left(p^{2}-m^{2}\right)\left(p \cdot \nabla \Sigma_{\mu \nu}-\left(F_{[\mu}^{\beta}+w_{[\mu}^{\beta}\right) \Sigma_{\nu] \beta}\right)=0 .
\end{gathered}
$$

By inserting (74) into (57) and employing the commutator $\left[\nabla_{\mu}, \nabla_{\nu}\right]=\left\{\left(\partial^{\beta} F_{\mu \nu}+\partial^{\beta} w_{\mu \nu}\right)-\left(F^{\beta \alpha}+w^{\beta \alpha}\right)\left(\partial_{p \alpha} \omega_{\mu \nu}\right)\right\} \partial_{p \beta}$, 
we obtain the following kinetic equation,

$$
\begin{aligned}
& \delta\left(p^{2}-m^{2}\right)\left[p \cdot \nabla f_{V}+\frac{\hbar}{4}\left\{\left(\partial^{\beta} F_{\mu \nu}+\partial^{\beta} w_{\mu \nu}\right)\right.\right. \\
& \left.-\left(F^{\beta \alpha}+w^{\beta \alpha}\right)\left(\partial_{p \alpha} \omega_{\mu \nu}\right)\right\} \Sigma^{\mu \nu} \partial_{p \beta} f_{A}^{0} \\
& \left.+\frac{\hbar}{4}\left\{\left(\partial^{\beta} F_{\mu \nu}+\partial^{\beta} w_{\mu \nu}\right)-\left(F^{\beta \alpha}+w^{\beta \alpha}\right)\left(\partial_{p \alpha} \omega_{\mu \nu}\right)\right\} f_{A}^{0} \partial_{p \beta} \Sigma^{\mu \nu}\right] \\
& -\frac{\hbar}{2} \delta^{\prime}\left(p^{2}-m^{2}\right)\left(F^{\alpha \beta}+w^{\alpha \beta}\right) \\
& \quad \times\left[\Sigma_{\alpha \beta} p \cdot \nabla f_{A}^{0}+f_{A}^{0} p \cdot \nabla \Sigma_{\alpha \beta}\right]=0
\end{aligned}
$$

where $f_{V} \equiv f_{V}^{0}+\hbar f_{V}^{1}$. We can express the last term of (80) in terms of the delta function, instead of its derivative: Let us multiply the zeroth-order equation (78) by $\left(F^{\alpha \beta}+w^{\alpha \beta}\right)$, use $\left(F^{\mu \nu}+w^{\mu \nu}\right)\left(F_{[\mu}^{\beta}+w_{[\mu}^{\beta}\right) \Sigma_{\nu] \beta}=0$, and then take its derivative with respect to momentum. Contracting the resultant equation with momentum results in

$$
\begin{gathered}
-\delta^{\prime}\left(p^{2}-m^{2}\right)\left(F^{\alpha \beta}+w^{\alpha \beta}\right) p \cdot \nabla \Sigma_{\alpha \beta} \\
=\frac{\delta\left(p^{2}-m^{2}\right)}{2 p^{2}}\left\{\left(F^{\alpha \beta}+w^{\alpha \beta}\right) p \cdot \nabla \Sigma_{\alpha \beta}\right. \\
\left.+p^{\mu} p^{\nu} \partial_{p \nu}\left[\left(F^{\alpha \beta}+w^{\alpha \beta}\right) \nabla_{\mu} \Sigma_{\alpha \beta}\right]\right\} .
\end{gathered}
$$

Thus the kinetic equation (80) can equivalently be written as

$$
\begin{aligned}
& \delta\left(p^{2}-m^{2}\right)\left\{p \cdot \nabla f_{V}+\frac{\hbar}{4}\left[\left(\partial^{\beta} F_{\mu \nu}+\partial^{\beta} w_{\mu \nu}\right)-\left(F^{\beta \alpha}+w^{\beta \alpha}\right)\left(\partial_{p \alpha} \omega_{\mu \nu}\right)\right] \Sigma^{\mu \nu} \partial_{p \beta} f_{A}^{0}+\frac{\hbar}{4}\left\{\left(\partial^{\beta} F_{\mu \nu}+\partial^{\beta} w_{\mu \nu}\right)\right.\right. \\
& \left.\left.-\left(F^{\beta \alpha}+w^{\beta \alpha}\right)\left(\partial_{p \alpha} \omega_{\mu \nu}\right)\right\} f_{A}^{0} \partial_{p \beta} \Sigma^{\mu \nu}+\frac{\hbar}{2 p^{2}} f_{A}^{0}\left(F^{\alpha \beta}+w^{\alpha \beta}\right) p \cdot \nabla \Sigma_{\alpha \beta}+\frac{\hbar p^{\mu} p^{\nu}}{2 p^{2}} f_{A}^{0} \partial_{p \nu}\left[\left(F^{\alpha \beta}+w^{\alpha \beta}\right) \nabla_{\mu} \Sigma_{\alpha \beta}\right]\right\} \\
& -\frac{\hbar}{2} \delta^{\prime}\left(p^{2}-m^{2}\right)\left(F^{\alpha \beta}+w^{\alpha \beta}\right) \Sigma_{\alpha \beta} p \cdot \nabla f_{A}^{0}=0 .
\end{aligned}
$$

There are four unknown functions in the general solutions of Wigner function components. Hence, we need to obtain some other kinetic equations: At the $\hbar^{2}$-order (61) yields

$$
p_{[\mu} \mathcal{V}_{\nu]}^{(2)}+\Pi_{[\mu}^{(2)} \mathcal{V}_{\nu]}^{(0)}+\frac{\hbar}{2} \epsilon_{\mu \nu \alpha \beta} \nabla^{\alpha} \mathcal{A}^{\beta(1)}=0
$$

$\mathcal{V}_{\nu}^{(2)}$ can be read from (53) and $\mathcal{A}_{\mu}^{(1)}$ is given by (75). Inserting them into (83) and using (77), (78), one finds

$$
\begin{aligned}
& \delta\left(p^{2}-m^{2}\right)\left[p \cdot \nabla\left(\Sigma_{\mu \nu} f_{A}^{0}+\hbar \Sigma_{\mu \nu}^{1}\right)\right. \\
& \left.-\left(w_{[\mu}^{\alpha}+F_{[\mu}^{\alpha}\right)\left(\Sigma_{\nu] \alpha} f_{A}^{0}+\hbar \Sigma_{\nu] \alpha}^{1}\right)+\frac{\hbar}{2}\left(\partial_{\alpha} F_{\mu \nu}+\partial_{\alpha} w_{\mu \nu}\right) \partial_{p}^{\alpha} f_{V}\right] \\
& -\hbar \delta^{\prime}\left(p^{2}-m^{2}\right)\left(F_{\mu \nu}+w_{\mu \nu}\right) p \cdot \nabla f_{V} \\
& +\hbar \delta^{\prime}\left(p^{2}-m^{2}\right) p^{\alpha}\left(F_{\alpha \rho}+w_{\alpha \rho}\right)\left(\partial_{p}^{\rho} w_{\mu \nu}\right) f_{V}=0
\end{aligned}
$$

By contracting it with $\frac{1}{2} \epsilon^{\mu \nu \alpha \beta} p_{\alpha},(84)$ can also be expressed as

$$
\begin{aligned}
& \frac{\delta\left(p^{2}-m^{2}\right)}{m}\left[p \cdot \nabla\left(s^{\beta} f_{A}^{0}\right)-\left(w^{\beta \alpha}+F^{\beta \alpha}\right) s_{\alpha} f_{A}^{0}\right] \\
& +\hbar \frac{\delta\left(p^{2}-m^{2}\right)}{2 p^{2}} \epsilon^{\mu \nu \alpha \beta} p_{\alpha}\left[p \cdot \nabla \Sigma_{\mu \nu}^{1}-\left(w_{[\mu}^{\alpha}+F_{[\mu}^{\alpha}\right) \Sigma_{\nu] \alpha}^{1}\right] \\
& +\hbar \frac{\delta\left(p^{2}-m^{2}\right)}{2 p^{2}} p_{\alpha}\left(\partial_{\sigma} \tilde{F}^{\alpha \beta}+\partial_{\sigma} \tilde{w}^{\alpha \beta}\right) \partial_{p}^{\sigma} f_{V} \\
& -\hbar \frac{\delta^{\prime}\left(p^{2}-m^{2}\right)}{p^{2}} p_{\alpha}\left(\tilde{F}^{\alpha \beta}+\tilde{w}^{\alpha \beta}\right) p \cdot \nabla f_{V} \\
& +\hbar \frac{\delta^{\prime}\left(p^{2}-m^{2}\right)}{p^{2}} p_{\alpha} p^{\sigma}\left(F_{\sigma \gamma}+w_{\sigma \gamma}\right)\left(\partial_{p}^{\gamma} \tilde{w}^{\alpha \beta}\right) f_{V}=0 .
\end{aligned}
$$

Let us focus on its projection in the spin quantization direction by multiplying it with $\left(-s^{\beta} / m\right)$, which is equivalent to taking contraction of (84) with $\frac{1}{2} \Sigma^{\mu \nu}$ :

$$
\begin{aligned}
& \delta\left(p^{2}-m^{2}\right)\left[p \cdot \nabla f_{A}+\frac{\hbar}{4} \Sigma^{\mu \nu}\left(\partial_{\alpha} F_{\mu \nu}+\partial_{\alpha} w_{\mu \nu}\right) \partial_{p}^{\alpha} f_{V}\right] \\
& -\frac{\hbar}{2} \delta^{\prime}\left(p^{2}-m^{2}\right) \Sigma^{\mu \nu}\left(F_{\mu \nu}+w_{\mu \nu}\right) p \cdot \nabla f_{V} \\
& +\frac{\hbar}{2} \delta^{\prime}\left(p^{2}-m^{2}\right) \Sigma^{\mu \nu} p^{\alpha}\left(F_{\alpha \rho}+w_{\alpha \rho}\right)\left(\partial_{p}^{\rho} w_{\mu \nu}\right) f_{V}=0
\end{aligned}
$$

where we introduced

$$
f_{A} \equiv f_{A}^{0}+\frac{\hbar}{2} \Sigma^{\mu \nu} \Sigma_{\mu \nu}^{1}
$$

By summing and subtracting (82) and (86) we get

$$
\begin{aligned}
& \delta\left(p^{2}-m^{2} \mp \frac{\hbar}{2} \Sigma_{\mu \nu}\left(F^{\mu \nu}+w^{\mu \nu}\right)\right)\left\{p \cdot \nabla\left(f_{V} \pm f_{A}\right)\right. \\
& \quad \pm \frac{\hbar}{4} \Sigma^{\mu \nu}\left(\partial_{x \alpha} F_{\mu \nu}+\partial_{x \alpha} w_{\mu \nu}\right) \partial_{p}^{\alpha}\left(f_{V} \pm f_{A}\right) \\
& \left.\quad-\frac{\hbar}{4} \Sigma^{\mu \nu}\left(F^{\beta \alpha}+w^{\beta \alpha}\right)\left(\partial_{p \alpha} w_{\mu \nu}\right) \partial_{p \beta} f_{A}\right\}+\mathcal{C}_{1} \pm \mathcal{C}_{2}=0 .
\end{aligned}
$$

$\mathcal{C}_{1}, \mathcal{C}_{2}$ designate the terms which do not contain derivatives of $f_{A}$ or $f_{V}$ : 


$$
\begin{aligned}
\mathcal{C}_{1}= & \delta\left(p^{2}-m^{2}\right) \frac{\hbar f_{A}}{4}\left\{\left\{\left(\partial^{\beta} F_{\mu \nu}+\partial^{\beta} w_{\mu \nu}\right)\right.\right. \\
& \left.-\left(F^{\beta \alpha}+w^{\beta \alpha}\right)\left(\partial_{p \alpha} \omega_{\mu \nu}\right)\right\} \partial_{p \beta} \Sigma^{\mu \nu} \\
& +\frac{2}{p^{2}}\left(F^{\alpha \beta}+w^{\alpha \beta}\right) p \cdot \nabla \Sigma_{\alpha \beta} \\
& \left.+\frac{2 p^{\mu} p^{\nu}}{p^{2}} \partial_{p \nu}\left[\left(F^{\alpha \beta}+w^{\alpha \beta}\right) \nabla_{\mu} \Sigma_{\alpha \beta}\right]\right\}, \\
\mathcal{C}_{2}= & \frac{\hbar}{2} \delta^{\prime}\left(p^{2}-m^{2}\right) \Sigma^{\mu \nu} p^{\alpha}\left(F_{\alpha \rho}+w_{\alpha \rho}\right)\left(\partial_{p}^{\rho} w_{\mu \nu}\right) f_{V} .
\end{aligned}
$$

The zeroth-order particle distribution functions given in (64), (65), can be extended as follows,

$$
\begin{aligned}
& \frac{1}{2}\left(f_{V}+f_{A}\right)=2 \sum_{e} \theta\left(e p^{0}\right) f_{R}^{e}(x, p), \\
& \frac{1}{2}\left(f_{V}-f_{A}\right)=2 \sum_{e} \theta\left(e p^{0}\right) f_{L}^{e}(x, p),
\end{aligned}
$$

where we relabeled $f_{1} \equiv f_{R}$ and $f_{-1} \equiv f_{L}$.

An alternative form of kinetic equations is given in Appendix.

\section{3D KINETIC EQUATIONS IN THE COMOVING FRAME}

In Sec. III, we modified the quantum kinetic equation in terms of $w_{\mu \nu}$ given in (47), where $v_{\mu}$ is an arbitrary frame velocity. Let us now deal with the comoving frame by setting $v_{\mu}=u_{\mu}$. As we have already mentioned, we consider vanishing linear acceleration, $u_{\nu} \partial^{\nu} u_{\mu}=0$. Let us choose the distribution function $f_{\chi}$ as

$$
f_{(u \cdot p) \chi}^{F D}=\sum_{e} \frac{2 \theta\left(e p^{0}\right)}{e^{e\left(u \cdot p-\mu_{\chi}\right) / T}+1} .
$$

Now one can observe that to satisfy the zeroth-order equations (76), (77), the fluid velocity should fulfill the condition $\partial_{\nu} u_{\mu}=-\partial_{\mu} u_{\nu}$. Therefore we can express the vorticity tensor as

$$
\partial_{\mu} u_{\nu}=\epsilon_{\mu \nu \rho \sigma} u^{\rho} \omega^{\sigma},
$$

where $\omega_{\mu}$ is the vorticity of fluid. The internal energy is $h=u \cdot p$. Thereby (47) can be written as follows

$$
\begin{aligned}
w_{\mu \nu}^{(C F)} & =u \cdot p \epsilon_{\mu \nu \sigma \rho} u^{\sigma} \omega^{\rho}+p^{\alpha}\left(u_{\nu} \epsilon_{\mu \alpha \sigma \rho}-u_{\mu} \epsilon_{\nu \alpha \sigma \rho}\right) u^{\sigma} \omega^{\rho} \\
& =\epsilon_{\mu \nu \sigma \rho} \omega^{\rho}\left\{2(u \cdot p) u^{\sigma}-p^{\sigma}\right\},
\end{aligned}
$$

where we employed the Schouten identity: $u_{\nu} \epsilon_{\mu \alpha \sigma \rho}=$ $-\left(u_{\mu} \epsilon_{\alpha \nu \sigma \rho}+u_{\alpha} \epsilon_{\nu \mu \sigma \rho}+u_{\sigma} \epsilon_{\rho \nu \mu \alpha}+u_{\rho} \epsilon_{\nu \mu \alpha \sigma}\right)$.
In order to obtain a 3D transport equation by integrating (88) over $p_{0}$, we need to specify $s^{\mu}$ in accord with the equation (78). Because of expressing it as in (66), this is equivalent to solve (78) for $\boldsymbol{n}_{e}$. Although the solution will be in the form $\boldsymbol{n}_{e}=\boldsymbol{n}_{e}(p, F, w)$, to have an idea about the 3D transport equations let us ignore its $F$ as well as $w$ dependence and choose it adequate to the helicity basis: $\boldsymbol{n}_{e}=\hat{\boldsymbol{p}}$. Obviously, 3D spin quantization direction for massive fermions need not to be in the direction of momentum. However, as we have seen in Sec. II, helicity basis is useful to obtain the mass corrections to the chiral effects. Therefore, the spin quantization direction fourvector becomes

$$
s_{e}^{\mu}(\boldsymbol{p}, \boldsymbol{p})=\left(\frac{|\boldsymbol{p}|}{m}, e \frac{E}{m} \hat{\boldsymbol{p}}\right) .
$$

Because of this choice when we integrate the kinetic equation (88) over $p_{0}$, we need to ignore the terms which are quadratic in fields. To perform the integral one should first solve the mass-shell condition for $p_{0}$ :

$$
p_{0}^{2}-E^{2}+\chi \hbar \frac{\epsilon^{\mu \nu \alpha \beta} p_{\alpha} s_{\beta}}{2 m}\left(F_{\mu \nu}+w_{\mu \nu}^{(C M)}\right)=0 .
$$

We work in the frame $u_{\mu}=(1, \mathbf{0}), \omega^{\mu}=(0, \boldsymbol{\omega})$, hence by substituting $s_{\mu}$ with (95), one can easily see that (96) dictates the dispersion relation

$$
p_{0}=e E-\frac{\hbar \chi}{2 E} \hat{\boldsymbol{p}} \cdot(\boldsymbol{B}+E \boldsymbol{\omega}) \equiv e \mathcal{E}_{e}^{\chi} .
$$

This is in accord with (28). Thus, we establish the 3D transport equation

$$
\left(\sqrt{\eta}{ }_{\chi}^{e} \frac{\partial}{\partial t}+(\sqrt{\eta} \dot{\boldsymbol{x}})_{\chi}^{e} \cdot \frac{\partial}{\partial \boldsymbol{x}}+(\sqrt{\eta} \dot{\boldsymbol{p}})_{\chi}^{e} \cdot \frac{\partial}{\partial \boldsymbol{p}}\right) f_{\chi}^{e}(t, \boldsymbol{x}, \boldsymbol{p})=0,
$$

where

$$
\begin{aligned}
\sqrt{\eta} \chi_{\chi}^{e} & =1-\frac{\hbar \chi}{2 m^{2}}\left(\hat{\boldsymbol{p}} \cdot \boldsymbol{B}-\frac{m^{2}+E^{2}}{E} e \hat{\boldsymbol{p}} \cdot \boldsymbol{\omega}\right), \\
(\sqrt{\eta} \dot{\boldsymbol{x}})_{\chi}^{e} & =\frac{\boldsymbol{p}}{E}-\hbar \chi \hat{\boldsymbol{p}}\left(\frac{\boldsymbol{p} \cdot \boldsymbol{B}}{2 E m^{2}}-\frac{\boldsymbol{p} \cdot \boldsymbol{B}}{2 E^{3}}+e \frac{\boldsymbol{p} \cdot \boldsymbol{\omega}}{2 m^{2}}\right), \\
(\sqrt{\eta} \dot{\boldsymbol{p}})_{\chi}^{e} & =e \boldsymbol{E}+2 \boldsymbol{p} \times \boldsymbol{\omega}+\frac{\boldsymbol{p} \times e \boldsymbol{B}}{E} .
\end{aligned}
$$

To acquire the vector and axial-vector currents, we insert (99) into the definition (31). Now the terms which are parallel to $\boldsymbol{B}$ and $\boldsymbol{\omega}$ can be shown to be

$$
\dot{j}_{A, V}^{B, \omega}(\boldsymbol{x}, t)=\bar{\sigma}_{A, V}^{B} \boldsymbol{B}+\bar{\sigma}_{A, V}^{\omega} \boldsymbol{\omega},
$$


with

$\bar{\sigma}_{A, V}^{B}=\frac{1}{6 \pi^{2} \hbar^{2}} \int d|\boldsymbol{p}|\left\{-\frac{|\boldsymbol{p}|^{5}}{m^{2} E^{3}} f_{A, V}^{F D}-\frac{|\boldsymbol{p}|^{3}}{E^{2}} \frac{\partial f_{A, V}^{F D}}{\partial E}\right\}$,

$\bar{\sigma}_{A, V}^{\omega}=\frac{1}{6 \pi^{2} \hbar^{2}} \int d|\boldsymbol{p}|\left\{-\frac{|\boldsymbol{p}|^{3}}{m^{2}} f_{A, V}^{F D}-\frac{|\boldsymbol{p}|^{3}}{E} \frac{\partial f_{A, V}^{F D}}{\partial E}\right\}$.

$f_{A, V}^{F D}$ are defined in (38). For $\mu_{R}=\mu_{L}=\mu$, at $T=0$, the vector current coefficients vanish and the axial-vector current coefficients can be calculated as

$$
\begin{array}{r}
\bar{\sigma}_{A}^{B}=\frac{1}{2 \pi^{2} \hbar^{2}}\left(\mu-\frac{\mu^{3}+8 m^{3}}{9 m^{2}}\right) \theta(\mu-m), \\
\bar{\sigma}_{A}^{\omega}=\frac{1}{4 \pi^{2} \hbar^{2}}\left(\mu^{2}-\frac{\mu^{4}}{6 m^{2}}-\frac{5 m^{2}}{2}\right) \theta(\mu-m) .
\end{array}
$$

These reflect the fact that in this formulation the zero mass limit cannot be achieved directly. In relativistic Wigner function formalism currents are calculated by integrating the equilibrium vector and axial-vector fields over the 4-momentum space. For massless fermions the chiral effects should not depend on the kinetic theory formalism. However, in the massive case equilibrium distributions depend on the formalism adopted to define the kinetic equations [29-32]. Hence there is no consensus about the mass corrections to chiral effects. Usually one discusses the small mass limit as we have done in Sec. II. However, the formalism which we adopted suits well with large mass [30]. Moreover, we have chosen the helicity basis (95) obtained by oversimplifying the kinetic equation (78). Nevertheless, this choice permitted us to show that our formalism yields the 3-dimensional kinetic theory with the correct dispersion relations (97) and the Coriolis force.

\section{DISCUSSIONS}

We studied the semiclassical kinetic theories of Dirac particles within two different approaches. First, the 3D formalism of Dirac particles [26], which manifestly exhibits the magnetic field, vorticity similarity is studied in helicity basis. We derived the axial-vector and vector currents for massive spin- $1 / 2$ particles. One of the distinguishing properties of this $3 \mathrm{D}$ formalism is the fact that massless limit can be reached effortlessly. Actually, one can easily observe that the currents (36), (37), generate the chiral magnetic and vortical effects correctly. We calculated the axial-vector current at zero temperature. In the small mass limit it is consistent with the Kubo formalism based calculations [39].

Dirac fermions in external electromagnetic fields can be considered as relativistic fluids described by the Wigner function satisfying the quantum kinetic equation which involves the electromagnetic field strength. For being able to take into account the noninertial characteristics of vorticity we modified this kinetic equation with terms depending on the four-velocity of rotating frame. We studied the equations satisfied by the Wigner function components and established the semiclassical relativistic kinetic equations of the scalar functions and spin degrees of freedom. Kinetic equations of the scalar functions are integrated over $p_{0}$ in a comoving frame by choosing the spin direction adequate to helicity basis. It is shown that the Coriolis force and the correct energy dispersion relation are generated. In addition, the coefficients of magnetic and vortical terms in axial-vector and vector currents are calculated at zero temperature. Unfortunately, the massless limit cannot be acquired directly within the approach which we have adopted to establish the semiclassical kinetic equations. Obviously, the resulting 3D model relies on the choice of spin quantization direction given in (95). For having a better understanding of 3D kinetic equations resulting from the relativistic ones, one should study the solution of (78) without ignoring the external electromagnetic fields and vorticity.

The original quantum kinetic equation was derived from the Dirac equation of charged particles coupled to the electromagnetic vector field $A_{\mu}(x)$. Hence, to consider the noninertial features of vorticity one can think to add a term proportional to the four-velocity $u_{\mu}(x)$ into the Dirac equation. It can be added only with a coefficient possessing the dimension of mass. Although internal energy, $h$, has mass dimension, in general it depends on momentum $p_{\mu}$. The unique possibility is to choose the coefficient equal to $m$. However, this choice does not yield an enthalpy current which we need to take into account the noninertial properties correctly. Hence, the modification which we propose does not seem to be generated by some gauge fieldlike terms coupled to Dirac particles.

\section{ACKNOWLEDGMENTS}

This work is supported by the Scientific and Technological Research Council of Turkey (TÜBİTAK) Grant No. 117F328.

\section{APPENDIX: ALTERNATIVE FORM OF KINETIC EQUATIONS}

All physical quantities are defined through integrals over momentum variables. Therefore, they are defined up to partial integrations. Consequently, we may get rid of the derivative of delta function in (86). First express it as $\frac{1}{2} \partial_{p}^{\alpha} \delta\left(p^{2}-m^{2}\right)=p^{\alpha} \delta^{\prime}\left(p^{2}-m^{2}\right)$, then perform the partial integration in the last term of (86), which results in

$$
-\frac{\hbar}{4} \delta\left(p^{2}-m^{2}\right)\left(F_{\alpha \beta}+w_{\alpha \beta}\right)\left(\partial_{p}^{\beta} w_{\mu \nu}\right)\left[\Sigma^{\mu \nu} \partial_{p}^{\alpha} f_{V}+\left(\partial_{p}^{\alpha} \Sigma^{\mu \nu}\right) f_{V}\right] .
$$

Thus (86) can equivalently be written as 


$$
\begin{gathered}
\delta\left(p^{2}-m^{2}\right)\left\{p \cdot \nabla f_{A}+\frac{\hbar}{4} \Sigma^{\mu \nu}\left(\partial_{\alpha} F_{\mu \nu}+\partial_{\alpha} w_{\mu \nu}\right) \partial_{p}^{\alpha} f_{V}-\frac{\hbar}{4}\left[\Sigma^{\mu \nu}\left(F_{\alpha \beta}+w_{\alpha \beta}\right)\left(\partial_{p}^{\beta} w_{\mu \nu}\right) \partial_{p}^{\alpha} f_{V}\right.\right. \\
\left.\left.+\left(F_{\alpha \beta}+w_{\alpha \beta}\right)\left(\partial_{p}^{\alpha} \Sigma^{\mu \nu}\right)\left(\partial_{p}^{\beta} w_{\mu \nu}\right) f_{V}\right]\right\}-\frac{\hbar}{2} \delta^{\prime}\left(p^{2}-m^{2}\right) \Sigma^{\mu \nu}\left(F_{\mu \nu}+w_{\mu \nu}\right) p \cdot \nabla f_{V}=0 .
\end{gathered}
$$

By summing and subtracting (82) and (A1) we get

$$
\begin{gathered}
\delta\left(p^{2}-m^{2} \mp \frac{\hbar}{2} \Sigma_{\mu \nu}\left(F^{\mu \nu}+w^{\mu \nu}\right)\right)\left\{p \cdot \nabla\left(f_{V} \pm f_{A}\right) \pm \frac{\hbar}{4} \Sigma^{\mu \nu}\left(\partial_{x \alpha} F_{\mu \nu}+\partial_{x \alpha} w_{\mu \nu}\right) \partial_{p}^{\alpha}\left(f_{V} \pm f_{A}\right)\right. \\
\left.\mp \frac{\hbar}{4} \Sigma^{\mu \nu}\left(F^{\beta \alpha}+w^{\beta \alpha}\right)\left(\partial_{p \alpha} w_{\mu \nu}\right) \partial_{p \beta}\left(f_{V} \pm f_{A}\right)\right\}+\tilde{\mathcal{C}}_{1} \pm \tilde{\mathcal{C}}_{2}=0 .
\end{gathered}
$$

$\tilde{\mathcal{C}}_{1}, \tilde{\mathcal{C}}_{2}$ indicate the terms which do not contain derivatives of $f_{A}$ or $f_{V}$ :

$$
\begin{aligned}
\tilde{\mathcal{C}}_{1}= & \delta\left(p^{2}-m^{2}\right) \frac{\hbar f_{A}}{4}\left\{\left\{\left(\partial^{\beta} F_{\mu \nu}+\partial^{\beta} w_{\mu \nu}\right)-\left(F^{\beta \alpha}+w^{\beta \alpha}\right)\left(\partial_{p \alpha} \omega_{\mu \nu}\right)\right\} \partial_{p \beta} \Sigma^{\mu \nu}\right. \\
& \left.+\frac{2}{p^{2}}\left(F^{\alpha \beta}+w^{\alpha \beta}\right) p \cdot \nabla \Sigma_{\alpha \beta}+\frac{2 p^{\mu} p^{\nu}}{p^{2}} \partial_{p \nu}\left[\left(F^{\alpha \beta}+w^{\alpha \beta}\right) \nabla_{\mu} \Sigma_{\alpha \beta}\right]\right\}, \\
\tilde{\mathcal{C}}_{2}= & -\frac{\hbar}{4} \delta\left(p^{2}-m^{2}\right)\left(F_{\alpha \beta}+w_{\alpha \beta}\right)\left(\partial_{p}^{\alpha} \Sigma^{\mu \nu}\right)\left(\partial_{p}^{\beta} w_{\mu \nu}\right) f_{V} .
\end{aligned}
$$

These kinetic equations do not involve derivatives of delta functions.

[1] D. E. Kharzeev, L. D. McLerran, and H. J. Warringa, The effects of topological charge change in heavy ion collisions: Event by event $\mathrm{P}$ and $C P$ violation, Nucl. Phys. A803, 227 (2008).

[2] K. Fukushima, D. E. Kharzeev, and H. J. Warringa, Chiral magnetic effect, Phys. Rev. D 78, 074033 (2008).

[3] D. Kharzeev and A. Zhitnitsky, Charge separation induced by P-odd bubbles in QCD matter, Nucl. Phys. A797, 67 (2007).

[4] M. A. Metlitski and A. R. Zhitnitsky, Anomalous axion interactions and topological currents in dense matter, Phys. Rev. D 72, 045011 (2005).

[5] K. Jensen, P. Kovtun, and A. Ritz, Chiral conductivities and effective field theory, J. High Energy Phys. 10 (2013) 186.

[6] D. T. Son and P. Surówka, Hydrodynamics with Triangle Anomalies, Phys. Rev. Lett. 103, 191601 (2009).

[7] Z. T. Liang and X. N. Wang, Globally Polarized QuarkGluon Plasma in Noncentral A + A Collisions, Phys. Rev. Lett. 94, 102301 (2005).

[8] F. Becattini, F. Piccinini, and J. Rizzo, Angular momentum conservation in heavy ion collisions at very high energy, Phys. Rev. C 77, 024906 (2008).

[9] J.-H. Gao, Z.-T. Liang, S. Pu, Q. Wang, and X.-N. Wang, Chiral Anomaly and Local Polarization Effect from the Quantum Kinetic Approach, Phys. Rev. Lett. 109, 232301 (2012).
[10] L. Adamczyk et al. (STAR Collaboration), Global $\Lambda$ hyperon polarization in nuclear collisions, Nature (London) 2017) 62 ,548).

[11] H. T. Elze, M. Gyulassy, and D. Vasak, Transport equations for the QCD quark Wigner operator, Nucl. Phys. B276, 706 (1986).

[12] D. Vasak, M. Gyulassy, and H. T. Elze, Quantum transport theory for abelian plasmas, Ann. Phys. (N.Y.) 173, 462 (1987).

[13] C. Y. Hidaka, S. Pu, and D. L. Yang, Relativistic chiral kinetic theory from quantum field theories, Phys. Rev. D 95, 091901 (2017).

[14] A. Huang, S. Shi, Y. Jiang, J. Liao, and P. Zhuang, Complete and consistent chiral transport from Wigner function formalism, Phys. Rev. D 98, 036010 (2018).

[15] B. J.-Y. Chen, D. T. Son, and M. A. Stephanov, Collisions in Chiral Kinetic Theory, Phys. Rev. Lett. 115, 021601 (2015).

[16] C. Y. Hidaka, S. Pu, and D. L. Yang, Nonlinear responses of chiral fluids from kinetic theory, Phys. Rev. D 97, 016004 (2018).

[17] Ö. F. Dayi and E. Kilinçarslan, Quantum kinetic equation in the rotating frame and chiral kinetic theory, Phys. Rev. D 98, 081701(R) (2018).

[18] I. Bialynicki-Birula, P. Gornicki, and J. Rafelski, Phasespace structure of the Dirac vacuum, Phys. Rev. D 44, 1825 (1991). 
[19] P. Zhuang and U. Heinz, Wigner functions in covariant and single-time formulations, Ann. Phys. (N.Y.) 266, 351 (1998).

[20] P. Zhuang and U. Heinz, Equal-time hierarchies for quantum transport theory, Phys. Rev. D 57, 6525 (1998).

[21] P. Zhuang and U. Heinz, Relativistic quantum transport theory for electrodynamics, Ann. Phys. (N.Y.) 245, 311 (1996).

[22] Ö. F. Dayi and E. Kilinçarslan, Some features of semiclassical chiral transport in rotating frames, Phys. Rev. D 100, 045012 (2019).

[23] Y.-C. Liu, L.-L. Gao, K. Mameda, and X.-G. Huang, Chiral kinetic theory in curved spacetime, Phys. Rev. D 99, 085014 (2019).

[24] J.-W. Chen, S. Pu, Q. Wang, and X.-N. Wang, Berry Curvature and Four-Dimensional Monopoles in the Relativistic Chiral Kinetic Equation, Phys. Rev. Lett. 110, 262301 (2013).

[25] J.-H. Gao, S. Pu, and Q. Wang, Covariant chiral kinetic equation in Wigner function approach, Phys. Rev. D 96, 016002 (2017).

[26] Ö. F. Dayi, E. Kilinçarslan, and E. Yunt, Semiclassical dynamics of Dirac and Weyl particles in rotating coordinates, Phys. Rev. D 95, 085005 (2017).

[27] M. A. Stephanov and Y. Yin, Chiral Kinetic Theory, Phys. Rev. Lett. 109, 162001 (2012).

[28] Ö. F. Dayi and E. Kilinçarslan, Nonlinear chiral plasma transport in rotating coordinates, Phys. Rev. D 96, 043514 (2017).

[29] J.-H. Gao and Z.-T. Liang, Relativistic quantum kinetic theory for massive fermions and spin effects, Phys. Rev. D 100, 056021 (2019).
[30] K. Hattori, Y. Hidaka, and D. L. Yang, Axial kinetic theory and spin transport for fermions with arbitrary mass, Phys. Rev. D 100, 096011 (2019).

[31] N. Weickgenannt, X.-L. Sheng, E. Speranza, Q. Wang, and D. H. Rischke, Kinetic theory for massive spin-1/2 particles from the Wigner-function formalism, Phys. Rev. D 100, 056018 (2019).

[32] Y.C. Liu, K. Mameda, and X. G. Huang, Covariant spin kinetic theory I: Collisionless limit, Chin. Phys. C 44, 094101 (2020).

[33] R.-H. Fang, L.-G. Pang, Q. Wang, and X.-N. Wang, Polarization of massive fermions in a vortical fluid, Phys. Rev. C 94, 024904 (2016).

[34] R.-H. Fang, L.-G. Pang, Q. Wang, and X.-N. Wang, Pseudoscalar condensation induced by chiral anomaly and vorticity for massive fermions, Phys. Rev. D 95, 014032 (2017).

[35] Z. Wang, X. Guo, S. Shi, and P. Zhuang, Mass correction to chiral kinetic equations, Phys. Rev. D 100, 014015 (2019).

[36] J.-Y. Chen, D. T. Son, M. A. Stephanov, H.-U. Yee, and Y. Yin, Lorentz Invariance in Chiral Kinetic Theory, Phys. Rev. Lett. 113, 182302 (2014).

[37] E. Gorbar, V. Miransky, I. Shovkovy, and X. Wang, Radiative corrections to chiral separation effect in QED, Phys. Rev. D 88, 025025 (2013).

[38] M. Buzzegoli, E. Grossi, and F. Becattini, General equilibrium second-order hydrodynamic coefficients for free quantum fields, J. High Energy Phys. 10 (2017) 091; Erratum, J. High Energy Phys. 07 (2018) 119.

[39] S. Lin and L. Yang, Mass correction to chiral vortical effect and chiral separation effect, Phys. Rev. D 98, 114022 (2018).

[40] W. Greiner and J. Reinhardt, Quantum Electrodynamics (Springer, Berlin, 2009). 